\title{
Glycan Microarray-Assisted Identification of IgG Subclass Targets in Schistosomiasis
}

\author{
Y. Y. Michelle Yang ${ }^{1}$, Angela van Diepen ${ }^{1}$, Katarzyna Brzezicka ${ }^{2}$, \\ Niels-Christian Reichardt ${ }^{2,3}$ and Cornelis H. Hokke ${ }^{1 *}$
}

${ }^{1}$ Department of Parasitology, Leiden University Medical Center, Leiden, Netherlands, ${ }^{2}$ Glycotechnology Laboratory, Centro de Investigación Cooperativa en Biomateriales (C/C biomaGUNE), San Sebastián, Spain, ${ }^{3}$ Centro de Investigación Biomédica en Red en Bioingeniería, Biomateriales y Nanomedicina (CIBER-BBN), San Sebastián, Spain

\section{OPEN ACCESS}

Edited by:

Thiago Almeida Pereira,

Stanford University, United States

Reviewed by:

Ricardo Riccio Oliveira Instituto Gonçalo Moniz (IGM), Fiocruz Bahia, Brazı

Daniel Claudio De Oliveira Gomes, Universidade Federal do Espírito Santo, Brazil

*Correspondence:

Cornelis H. Hokke

C.H.Hokke@/umc.n

Specialty section:

This article was submitted to

Microbial Immunology,

a section of the journa

Frontiers in Immunology

Received: 13 July 2018 Accepted: 19 September 2018 Published: 09 October 2018

Citation: Yang YYM, van Diepen A, Brzezicka K, Reichardt N-C and Hokke CH (2018)

Glycan Microarray-Assisted Identification of IgG Subclass Targets in Schistosomiasis.

Front. Immunol. 9:2331 doi: 10.3389/fimmu.2018.02331
Infection with schistosomes is accompanied by the induction of antibodies against the parasite. Despite having IgG against both protein and glycan antigens, infected individuals remain chronically infected until treated, and re-infection is common in endemic areas as immunity does not develop effectively. Parasite specific lgG subclasses may differ in functionality and effectivity with respect to effector functions that contribute to parasite killing and immunity. In this study, we investigated if specific IgG subclasses target specific antigenic schistosome glycan motifs during human infection. Sera from $41 \mathrm{~S}$. mansoni infected individuals from an endemic area in Uganda were incubated on two glycan microarrays, one consisting of a large repertoire of schistosome glycoprotein- and glycolipid- derived glycans and the other consisting of chemically synthesized core xylosylated and fucosylated N-glycans also expressed by schistosomes. Our results show that highly antigenic glycan motifs, such as multi-fucosylated terminal GalNAc( $\beta 1-4)$ GlcNAc (LDN) can be recognized by all IgG subclasses of infection sera, however with highly variable intensities. Detailed examination of core-modified $\mathrm{N}$-glycan targets revealed individual antibody responses specific for core-xylosylated and core $\alpha 3$-fucosylated glycan motifs that are life stage specifically expressed by schistosomes. IgG1 and IgG3 were detected against a range of $\mathrm{N}$-glycan core structures, but IgG2 and IgG4, when present, were specific for the core $\alpha 3$-fucose and xylose motifs that were previously found to be $\lg E$ targets in schistosomiasis, and in allergies. This study is the first to address Ig G subclass responses to defined helminth glycans.

\section{Keywords: glycan antigens, schistosomiasis, IgG subclass, glycan-microarray, fucosylation}

\section{INTRODUCTION}

Schistosomiasis is a parasitic infection of humans and mammals contracted by exposure to schistosome cercariae shed by infected aquatic snails. Once the parasite establishes itself in a human host, the infection which is associated with debilitating pathology due to tissue-deposition of eggs remains chronic until treated. In the schistosomiasis endemic areas in Africa, South-America, and South-East Asia, reinfection after treatment is common. Longitudinal studies have shown that resistance to re-infection with schistosomes develops very slowly. Many years of exposure to schistosomes and multiple treatments are required for the immune response to 
become effective (1-3). Various studies have shown that antischistosome antibodies are pivotal for anti-parasite immunity. Passive immunization with serum or monoclonal antibodies against schistosome antigens (4-7) were shown to reduce worm burden and egg production in previously unexposed mice upon challenge with schistosomes. Moreover, transfer of sera and of purified IgG from animals immunized with the protective Smp80 antigen conferred resistance to challenge infection, and it has been shown that the level of protection induced by immunization with Sm-p80 is reduced in antibody deficient mice (8).

As schistosomes are highly glycosylated organisms that express many glycan motifs different from mammals, it is not surprising that an abundance of antibodies are generated against schistosomal protein- and lipid-linked glycans exposed to the host (9-14). It is becoming increasingly clear that glycan antigens play an important role in helminth infection immunology, but it remains ambiguous whether anti-glycan antibodies contribute positively or negatively to protection.

Previously we have studied the anti-glycan IgG and IgM responses in schistosome infected rhesus macaques that are able to expel the worms (15), in order to identify glycan targets of antibodies that might be involved in the self-cure mechanism. We showed that serum IgG antibodies against highly fucosylated schistosome glycoproteins and glycolipids formed during infection were sustained when the macaques cleared the infection, indicating that these antibodies are present in a protective context and possibly play a role in the killing of adult worms in the host. Additionally, sera from macaques containing high titres of IgG against highly fucosylated motifs were able to kill schistosomula in vitro (15) and it has been shown that a monoclonal antibody specific for the fucosylated LeX antigen was protective in a mouse model of S. mansoni infection (6). On the other hand, humans that are generally susceptible to reinfection also have high titres of IgG antibodies against highly fucosylated motifs (16), indicating that anti-glycan antibodies are not necessarily protective. Indeed, some studies have regarded schistosome glycans to form a smokescreen that diverts the host immune system from attacking vulnerable peptide epitopes that could mediate protective responses (17-19). It has however been appreciated that protective glycan epitopes may also occur, but these could be under-represented and masked by irrelevant or "smokescreen" epitopes (20). As a consequence, protective antischistosome glycan responses may be difficult to identify. These observations called for investigations into differential recognition of specific schistosome glycan motifs in different models and cohorts such as reported in several recent glycan microarrayassisted studies $(12-16,21)$.

Alternatively, research into the IgG subclass-specific response toward defined glycan antigens may shed light on the role of anti-glycan antibodies in infection. Perhaps the key to associate glycan antigens with immunity is not in the antigen itself, but rather by the type of antibody response generated. Particular IgG subclasses have been found to correlate with resistance or susceptibility to schistosome infection: IgG1 against schistosome surface antigens were observed in putatively resistant individuals in a Brazilian cohort (22), while individuals that were chronically infected lacked high IgG1 against the same antigens, but mounted high IgG4 toward various schistosome antigens instead. IgG4, as well as IgG2 have been found to correlate with susceptibility of schistosome reinfection $(23,24)$. On the other hand, human serum IgG1 and IgG3, as well as IgE have been found to be potent inducers of eosinophilmediated cytotoxicity, while IgG4 antibodies were found to be inhibiting cytotoxicity (25) and compete with IgE to prevent antigen cross-linking and IgE mediated effector function (26). So far, studies on IgG subclass response against schistosome antigens have focused on schistosome surface proteins (22) or complex ill-defined schistosome antigen mixtures such as SEA or AWA (27). Little is known however about IgG subclass reactivity to defined schistosome glycan antigens. Interestingly, a recent study has shown that anti-glycan $\operatorname{IgE}$ responses are highly restricted toward only a few specific epitopes out of the many glycan antigens expressed by schistosomes, in particular N-glycan core-xylose or core $\alpha 3$ fucose, motifs that are often associated with plant glycans and allergens (28).

In the current study, we address the question whether specific serum IgG subclass responses to defined antigenic glycans occur during human schistosome infection, and whether these might be restricted to certain motifs or not. To this end, we tested the IgG subclass responses in a selection of 41 serum samples from a relatively homogeneous population in terms of exposure and infection intensity $(16,29)$ toward a large collection of schistosome glycan antigens. Using two glycan microarrays, one comprising a large set of native glycans isolated from different schistosome life-stages (14-16), the other comprising a set of synthetic $\mathrm{N}$-glycans with different core and branch modifications previously described (21) we determined for each of the IgG subclasses which glycan motifs are targeted during infection, and evaluated whether these are different or not for IgG1-4. Our results indicate that in schistosomiasis sera, each of the IgG subclasses can be directed against a variety of antigenic glycan motifs, but with different patterns for the different individuals tested. To our knowledge, this is the first study that addresses IgG subclass responses to defined glycans and glycan motifs in a helminth infection.

\section{MATERIALS AND METHODS}

\section{Sera}

Sera used in this study were described in an earlier publication (16) where 41 schistosome infected individuals were selected from an original cohort study in a highly prevalent $S$. mansoni endemic area (29). The cohort study obtained ethical approval from the Uganda National Council for Science and Technology (UNCST) and was supported by the Cambridge Local Research Ethics Committee. Selected subjects had an age range of 5 to 46 years old, all with patent $S$. mansoni infection. The geometric mean of the intensity of infection measured by egg per gram feces was $560.65\left(\mathrm{CI}_{95 \%}\right.$ : 343.88, 914.05). 


\section{Glycan Microarray Construction And Incubation}

A glycan microarray constructed of glycans derived from $S$. mansoni cercariae (75 N-glycan fractions and $102 \mathrm{O}$-glycan fractions), adult worms (77 N-glycan fractions and $31 \mathrm{O}$-glycan fractions) and eggs (57 egg N-glycan fractions and 98 soluble egg antigen $\mathrm{O}$-glycan fractions), and 30 glycans derived from glycosphingolipids representing multiple life stage, was described previously $(14,16)$. Twenty-four blank spots with spot buffer were included for array background control. Each glycan fraction was immobilized on a glass slide in triplicate. The synthetic glycan microarray containing a collection of core-xylosylated and core- $\alpha 3$ and $-\alpha 6$ fucosylated N-glycans with various core extensions has been described previously (21).

Binding assays of individual or pooled sera on both arrays were conducted following the protocol as described previously (14-16). Briefly, the microarray slide was blocked with $2 \%$ BSA, $50 \mathrm{mM}$ ethanolamine in PBS. Serum samples were diluted 1:100 in PBS-0.01\% Tween 20 with 1\% BSA. All four mouse anti-human isotype antibodies were purchased from Invitrogen. IgG1 and IgG3 were labeled with the Promokine PF-647 labeling kit; IgG2 and IgG4 were labeled with the PF-555 labeling kit following the manufacturer's protocol. All anti-human IgG subclass antibodies were diluted 1:200 in PBS-0.01 Tween20 to detect bound serum antibodies on the slide. All washing steps were performed with successive rinses with PBS-0.05\% Tween 20 and with PBS. The last washing step was finished by an additional wash with milliQ water and the slides were dried and kept in the dark until scanning.

\section{Scanning And Data Analysis}

A G2565BA scanner (Agilent Technologies, Santa Clara, CA) was used to scan the slides for fluorescence at $10 \mu \mathrm{m}$ resolution using lasers at 532 and $633 \mathrm{~nm}$. Anti-IgG2 and anti-IgG4 antibodies were detected at $532 \mathrm{~nm}$ and anti-IgG1 and anti-IgG3 antibodies at $633 \mathrm{~nm}$. Data and image analysis was performed with GenePix Pro 7.0 software (Molecular Devices, Sunnyvale, CA). Spots were aligned and re-sized using round features with no CPI threshold. Background-subtracted median intensities were averaged per time point and processed as described by Oyelaran et al. (30). Datasets were $\log _{2}$ transformed to remove the basic trends of variance. A hierarchical clustering analysis (HCA, complete linkage clustering using Euclidean distance metric) was performed to group associated glycan fractions using MultiExperiment Viewer v4.5.

\section{RESULTS}

\section{Serum IgG Subclass Response Against Glycans Expressed by Schistosomes in Infected Individuals}

To investigate whether IgG subclasses in sera from a schistosomeinfected cohort react with specific motifs or subsets of parasiteassociated glycans, we determined IgG1, IgG2, IgG3. and IgG4 binding intensities against a large variety of glycans in pooled sera of 41 infected individuals using glycan microarrays. These sera were from a relatively homogeneous population in terms of exposure and infection intensity). All individuals had patent S. mansoni infection (geometric mean infection intensity (epg) was $560.65\left(\mathrm{CI}_{95 \%}\right.$ : $\left.343.88,914.05\right)$. We found that in the pool IgG of all four subclasses are present against a wide range of schistosome-derived N-, O-, and glycosphingolipid glycans printed on the array (Figure 1A). IgG1 and IgG2 bound to the various glycans with similar high intensities, while antiglycan IgG4 binding intensities were lowest out of the four subclasses. Glycan targets for each of the IgG subclasses consisted mainly of highly fucosylated LDN epitopes, abundantly expressed on glycolipid-derived glycans and cercarial O-glycans (31). Interestingly, IgG subclass binding of $\mathrm{N}$-glycan core xylose (abundant on glycans from cercariae and miracidia) and core $\alpha 3$ fucose epitopes (abundant on glycans from eggs and miracidia), as determined by use of the synthetic glycan array, were rather different across the four subclasses (Figure 1B). IgG1, IgG3, and IgG4 in pooled infection serum showed higher binding intensities to core $\alpha 3$-fucose containing structures than to structures containing only core-xylose, with IgG4 being restricted to different subsets of the $\alpha 3$-fucose containing structures. In contrast, IgG2 response in this pool was mainly directed toward core xylose containing $\mathrm{N}$-glycans. Core $\alpha 3$-fucose has been shown to be an antigenic target in schistosome-infected humans, mice and rhesus macaques $(12,15)$. Interestingly, alone or in combination with core xylose, core $\alpha 3$-fucose also forms the major cross-reactive carbohydrate epitopes that are IgE targets on a variety of plant-derived allergens $(28,32,33)$. To further analyse the differential reaction of specific IgG subclasses with core-modified $\mathrm{N}$-glycans, we investigated the IgG subclass response in each infected individual on the synthetic glycan array.

\section{Schistosome Infected Individuals Differentially Express IgG Subclass Antibodies Toward Schistosome Glycans}

Among the individual sera incubated on the synthetic glycan array a striking variability in IgG subclass response toward the core-modified glycans was observed (Figure S1). Most sera contained antibodies binding to core $\alpha 3$-fucose and core xylose modified $\mathrm{N}$-glycans. However, this was not a universal response and no clear correlation between IgG subclass and glycan motifs was observed.

\section{IgG Subclass Response Profile of Schistosome Infected Individuals}

In view of the variations in IgG subclass responses to the core-modified $\mathrm{N}$-glycans between individuals, we performed a hierarchical clustering analysis and grouped the individuals based on response patterns for each IgG subclass (Figure 2A). We observed a cluster of individuals that had specific and high IgG1, 2, 3, or 4 binding to core $\alpha 3$-fucose (cluster red). Another cluster of individuals had high IgG binding to core xylose containing structures (cluster blue), while the third cluster, with the highest number of individuals, only had lower amounts of IgG binding to either core $\alpha 3$-fucose or 
A
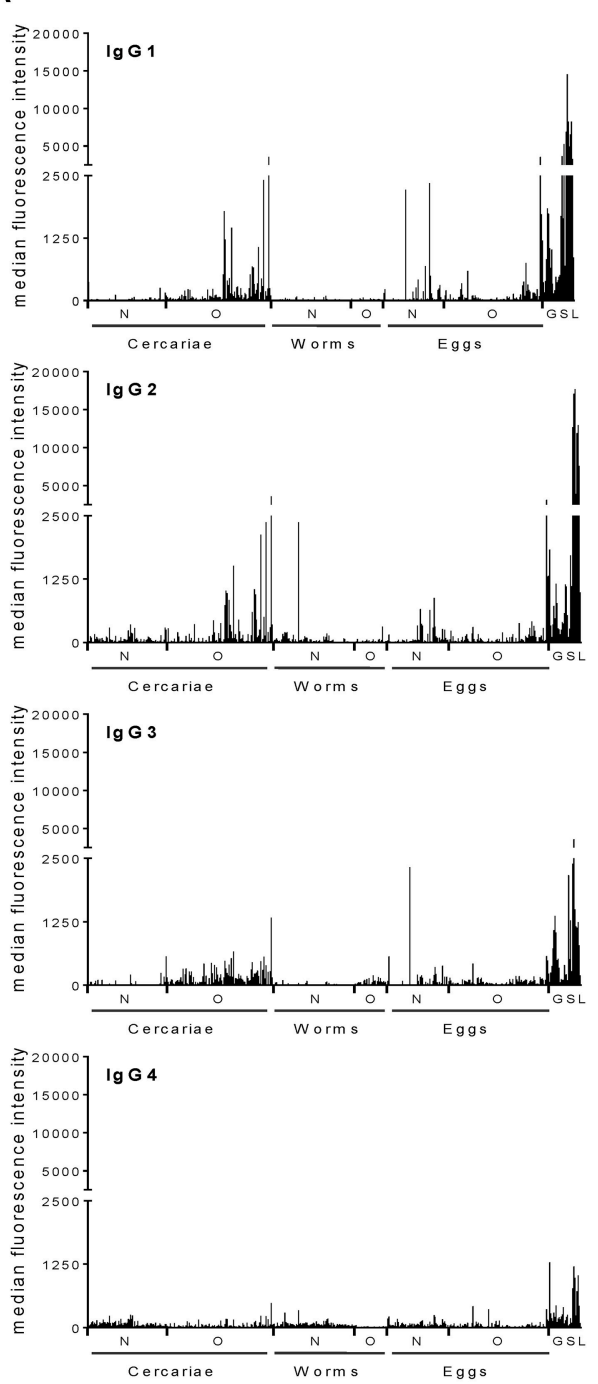

B
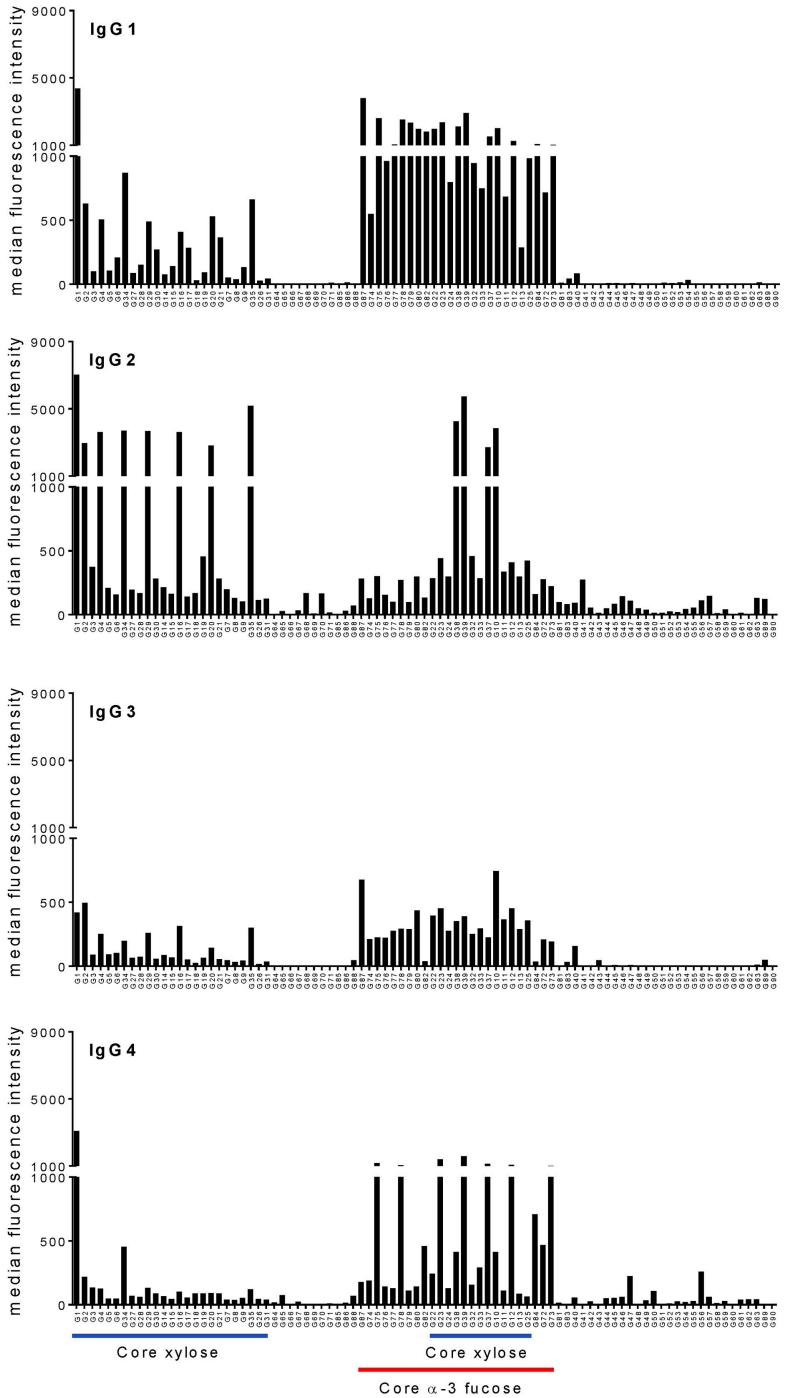

FIGURE 1 | Schistosome infected individuals produce IgG subclass antibodies to schistosome glycans. Averaged serum IgG subclass response from schistosome-infected individuals to (A) complex N-, O-, and glycoshingolipid glycans isolated from different life stages of schistosomes and (B) core modified $\mathrm{N}$-glycans synthesized chemically. The horizontal axis indicates different glycan structures. Each bar corresponds to antibody binding to individual glycan fractions printed on the glycan microarray. Schistosome GSL glycans are shown as a group irrespective of the life stage. N, N-glycans; O, O-glycans; GSL, Glycoshingolipid derived glycans.

core xylose (cluster yellow). When comparing the IgG subclass distribution for each motif within each serum, we saw that some individuals had high binding to core $\alpha 3$-fucose with all four IgG subclasses (cluster red in all subclasses) (Figure 2B), while other individuals responded strongly to core $\alpha 3$-fucose structures with only one or two subclasses that were variably of the IgG1, 2, 3, or 4 type. Similarly, two individuals of the 41 individuals responded strongly to core xylose containing $\mathrm{N}$ glycans with all IgG subclasses (cluster blue in all subclasses), while other individuals responded only with one or two subclasses, variably of the IgG2, IgG3, or IgG4 class. These observations indicate that IgG subclass responses toward these glycan antigens are not restricted by the nature of the antigen, but rather appear to be related to each individual response to the infection. One interesting observation was that most of the individuals that had high IgG subclass response toward either core xylose or core $\alpha 3$-fucose were children aged 12 or younger (Figure 2B).

\section{Structural Motifs Bound by IgG1-4}

Next, to gain more insight in the structural determinants that are recognized specifically by the different IgG subclasses, we examined in detail how the binding of IgG1, IgG2, IgG3, and IgG4 to core $\alpha 3$-fucose and core xylose is influenced by 


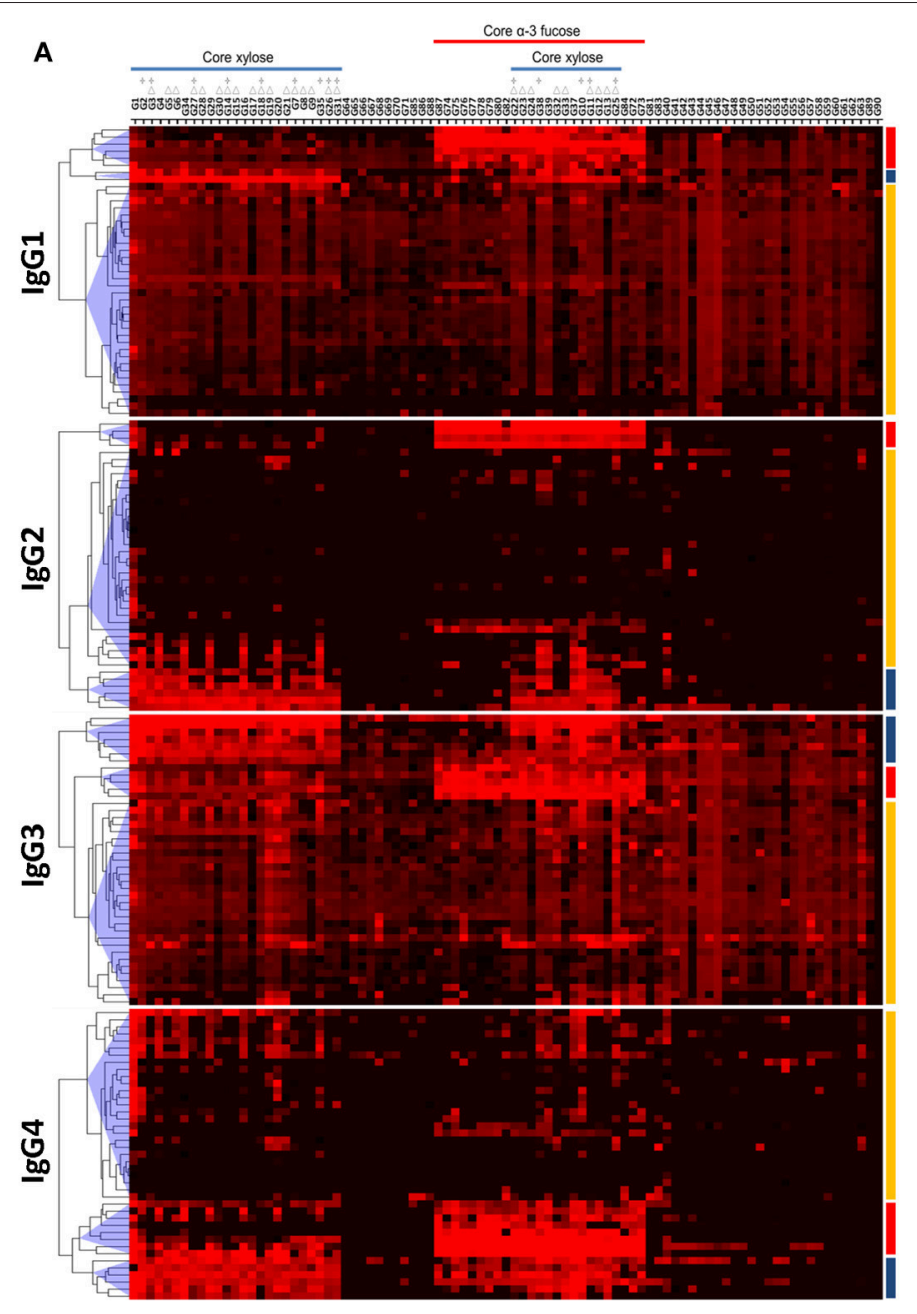

B

\begin{tabular}{|c|c|c|c|c|}
\hline \multirow{2}{*}{ - $\$ 41$} & IgG1 & IgG2 & $\operatorname{lgG} 3$ & $\operatorname{lgG} 4$ \\
\hline & & & & \\
\hline \multicolumn{5}{|l|}{$\bullet S 22$} \\
\hline \multicolumn{5}{|l|}{ • S21 } \\
\hline \multicolumn{5}{|l|}{ - $\$ 26$} \\
\hline \multicolumn{5}{|l|}{ - S31 } \\
\hline \multicolumn{5}{|l|}{ OS17 } \\
\hline \multicolumn{5}{|l|}{ - S27 } \\
\hline \multicolumn{5}{|l|}{ OS12 } \\
\hline \multicolumn{5}{|l|}{ - $\$ 23$} \\
\hline \multicolumn{5}{|l|}{ - $\$ 30$} \\
\hline \multicolumn{5}{|l|}{$S 11$} \\
\hline \multicolumn{5}{|l|}{$S 10$} \\
\hline \multicolumn{5}{|l|}{057} \\
\hline \multicolumn{5}{|l|}{ - $\$ 34$} \\
\hline \multicolumn{5}{|l|}{$\$ S 15$} \\
\hline \multicolumn{5}{|l|}{ - $\mathrm{S} 32$} \\
\hline \multicolumn{5}{|l|}{ - $\$ 25$} \\
\hline \multicolumn{5}{|l|}{ - $\$ 29$} \\
\hline \multicolumn{5}{|l|}{54} \\
\hline \multicolumn{5}{|l|}{056} \\
\hline \multicolumn{5}{|l|}{516} \\
\hline \multicolumn{5}{|l|}{ - 539} \\
\hline \multicolumn{5}{|l|}{ S18 } \\
\hline \multicolumn{5}{|l|}{$0 S 8$} \\
\hline \multicolumn{5}{|l|}{$\mathrm{S} 1$} \\
\hline \multicolumn{5}{|l|}{53} \\
\hline - $\$ 36$ & & & & \\
\hline ○5 & & & & \\
\hline o S2 & & & & \\
\hline - $\$ 33$ & & & & \\
\hline - $\$ 37$ & & & & \\
\hline - \$24 & & & & \\
\hline o 59 & & & & \\
\hline - $\$ 28$ & & & & \\
\hline $0 \$ 20$ & & & & \\
\hline - 538 & & & & \\
\hline$\$ 19$ & & & & \\
\hline - 540 & & & & \\
\hline - S35 & & & & \\
\hline OS14 & & & & \\
\hline o $\$ 13$ & & & & \\
\hline
\end{tabular}

FIGURE 2 | Hierarchical clustering analysis of anti-glycan antibody responses in schistosome infected individuals. (A) Heatmap showing lgG subclass response of schistosome infected subjects (columns) to core modified N-glycan fractions (rows) that have been synthesized and described by Brzezicka et al. Median fluorescence intensity was corrected for baseline and $\log _{2}$ transformed; increase in antibody binding is indicated by the red color intensity. Three major clusters of subjects were identified for each lgG subclass based on antibody binding intensity to glycans: one cluster of individuals had high lgG binding to core $\alpha 3$-fucose (red), another cluster of individuals to core xylose (blue), and one cluster of individuals without specific binding to core $\alpha 3$-fucose or core xylose (yellow). Core xylosylated and core $\alpha-3$ fucosylated structures are indicated. Within core-xylose containing structures, those that have additional monosaccharides on the $\alpha$-3mannose $(\Delta)$ and those that miss the core $\alpha-6$ mannose $\left(^{*}\right)$ are indicated. (B) IgG subclass response profile of each schistosome infected individual. Red: high response against core a3-fucose; blue: high response against core xylose; yellow: low response against other glycans. Filled circles (•): age 12 and under. Open circles (०): age 20 and older.

adjacent structural elements. Comparison of the signal intensities of four sets of structurally related synthetic glycan structures first revealed that moderate levels of IgG1 and IgG3 reactive with the unsubstituted trimannosyl core glycan $\left(\mathrm{Man}_{3} \mathrm{GlcNAc}_{2}\right)$ (G42) were detected in most sera tested (Figures 3A-C). In contrast, IgG2 and IgG4 to the N-glycan core were only detected when either a xylose (G34) or an $\alpha 3$-fucose (G73) residue were present (Figures 3A,C). Interestingly, IgG1 and IgG3 in general did not become more reactive upon addition of core xylose to the trimannosyl core, but signal intensities did increase significantly upon $\alpha 3$-fucosylation while core $\alpha 6$-fucose addition (G64) had the opposite effect of lowering the IgG1 and IgG3 response to the otherwise unsubstituted trimannosyl core. The addition of $\alpha 6$-fucose had no effect on the reactivity of any of the four IgG subclasses to the $\alpha 3$-fucosylated core however (G64) (Figure 3C). Regarding the combination of both the antigenic core xylose and core $\alpha 3$-fucose modifications it appears that sera where IgG2 and IgG4 reactivity is negative against core $\alpha 3$-fucose becomes positive upon addition of the xylose (G37) (Figure 3D). Since reactivity is also observed to core-xylosylated glycans without fucose, it appears that $\alpha 3$ fucose and xylose form independent antigenic motifs. Finally, IgG2 and IgG4, but not IgG1 and IgG3 reactivity against core xylose are hindered by the addition of a GlcNAc branch to the 3-linked Man (G5), but removal of the 3-linked Man (G1) enhances reactivity, indicating that reactivity to xylose depends on its spatial accessibility (Figures 3A,B). Together these data suggested that IgG2 and IgG4 reactivity to N-glycan core 


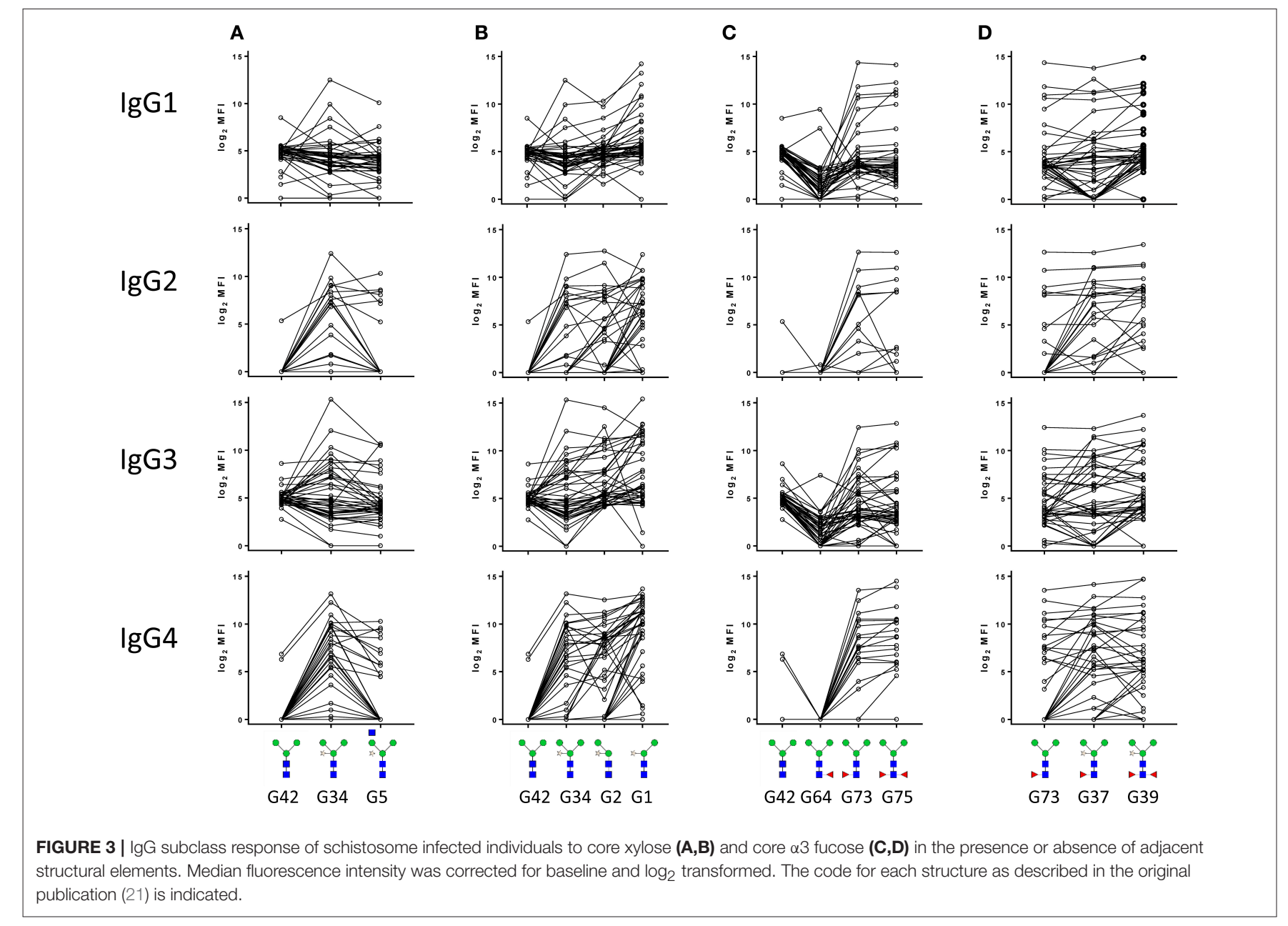

structures in schistosomiasis sera are either against the core xylose and core $\alpha 3$-fucose motifs, whereas IgG1 and IgG3 are less specific and restricted.

\section{DISCUSSION}

In this study we have investigated the IgG subclass responses against defined parasite-derived glycans in schistosome-infected individuals. Detailed glycan microarray analysis indicated that IgG1, 2, 3, and 4 in schistosome infection sera are variably present against a wide range of antigenic parasite glycans. Interestingly, individuals infected with schistosomes respond with particularly high variability to the antigenic $\alpha 3$-fucose and $\beta 2$-xylose core modifications of $\mathrm{N}$-glycans with respect to the different IgG subclasses. This variability in antibody response was not a reflection of the intensity of infection as all sera included this study were from heavily infected individuals living in a schistosome endemic area. The sera used in this study were selected from a larger study (29), where selected individuals had an age range between 5 to 46 years old, all with patent S. mansoni infection, with fecal egg counts between 343.88 and 914.05 epg $\left(\mathrm{CI}_{95 \%}\right.$ of geometric mean). Previously, we have used this set of sera to compare the anti-glycan (total) IgG and IgM in schistosome infected children and adults (16) and found that although there were anti-glycan antibody differences between children and adults, other factors apart from age also played a role in shaping antiglycan antibody response profiles. It is well-known that the overall serum IgG subclass distribution is generally different between adults and children. IgG1 and IgG3 development is faster and reaches around $75 \%$ of the adult serum levels at the age of five, while IgG2 and IgG4 levels rise much slower, reaching $70 \%$ of adult serum levels at the age of 14 . Nevertheless, large inter-individual variations were observed previously for IgG subclass development (34), as corroborated by our study. Although most individuals with responses of any subclass toward core modified glycans were children, many children did not produce antibodies against core modified N-glycans, raising the question which other factors, possibly cross-reactive antigens, determine the induction of these schistosome-reactive antibodies.

The sample selection used here to study the glycan specificity of IgG subclasses in schistosomiasis infection serum is not suitable for addressing immunoepidemiological questions. 
Nonetheless, the variable subclass responses to specific glycan antigens observed in this study may have the potential to reflect the immune profile of infected individuals and act as markers for infection with respect to intensity, exposure, chronicity, immunopathology or resistance to infection. High IgG1 titers against a specific set of schistosome tegument protein antigens for instance have been observed in putatively resistant individuals in a Brazilian cohort but not in chronically infected individuals, where high IgG4 antibodies against the same schistosome antigens were observed instead (22). In addition, IgG1 and IgG3 against protective schistosome antigens has also been found in naturally resistant individuals but not in chronically infected or unexposed individuals (35). These studies indicate the importance of IgG1 against protective antigens in developing immunity. In contrast, susceptibility to reinfection in humans has been associated with high levels of IgM, IgG2, and IgG4 (23, $24,26,27)$. The presence of IgM, IgG2, and IgG4, purified from serum of infected individuals, prevented eosinophil mediated killing of schistosomula by other IgG subclasses present in human infection sera (25). The IgG4 subclass is usually produced after repeated, long-term antigenic stimulation, and has minimal effector functions. Literature describes highly elevated IgG4 toward schistosome antigens (26) that may compete with IgE and prevent antigen cross-linking and $\operatorname{IgE}$ mediated effector functions. Interestingly, although we have not observed very high IgG4 binding intensities against schistosome glycans, precisely the core $\alpha 3$-fucose and core xylose motifs that form the cross-reactive carbohydrate determinants for $\operatorname{IgE}$ in plant allergens as well as helminths $(28,33,36)$ are also the only IgG4 reactive glycan elements in the tested N-glycans in the current study. It should be noted that expression of core $\alpha 3$ fucose and core xylose motifs during the schistosome life cycles appears to be highly specific. By mass spectrometric glycomics approaches it has been shown that core xylose is abundant on $\mathrm{N}$-glycans of cercariae and miracidia (31), whereas core $\alpha 3$ fucose has been detected in abundance in N-glycans on the secretory egg glycoproteins IPSE/ $\alpha 1$ (37) and omega-1 (together with core $\alpha 6$-fucose) (38) and on $\mathrm{N}$-glycans derived from the miracidia (31) and the egg glycoprotein kappa-5 (together with $\alpha 6$-fucose and core xylose) (39). Both core xylose and core $\alpha 3$-fucose are expressed by plant glycoproteins $(33,40)$ and in a small subset of other helminths (36). The specific IgG determined in our study may therefore also have been

\section{REFERENCES}

1. Butterworth AE, Fulford AJ, Dunne DW, Ouma JH, Sturrock RF. Longitudinal studies on human schistosomiasis. Philos Trans R Soc Lond B Biol Sci. (1988) 321:495-511.

2. Fulford AJ, Butterworth AE, Sturrock RF, Ouma JH. On the use of ageintensity data to detect immunity to parasitic infections, with special reference to Schistosoma mansoni in Kenya. Parasitology (1992) 105 (Pt 2):21 9-27.

3. Vereecken K, Naus CW, Polman K, Scott JT, Diop M, Gryseels B, et al. Associations between specific antibody responses and resistance to reinfection in a Senegalese population recently exposed to Schistosoma mansoni. triggered by other antigen sources other than schistosomes. It would be interesting to investigate the IgG subclass response of infected individuals toward more complex glycan antigens that are schistosome specific, such as highly fucosylated LDN epitopes on glycoproteins and glycosphingolipids of the parasite and its secretions. Such complex schistosome antigens, induce higher total IgG binding in schistosome infected rhesus macaques compared to core modified epitopes (15). Given that highly fucosylated motif such as Fuca1-2Fuca1-3 on LDN have not been found in organisms other than schistosomes, it would be interesting to see if different individuals would have a less variable IgG subclass distribution to these complex, more parasite-specific glycan antigens, when these would become available as synthetic, defined antigens.

In this study we have for the first time investigated the IgG subclass response against schistosome glycans in infected individuals. The complex interplay between how antigens trigger IgG subclass response calls for analysis with a larger human cohort with better defined resistant and susceptible immune profiles, to be able to understand whether particular subclasses against particular glycan epitopes are associated with a disease state. Although high interindividual antibody variation was observed against core modified $\mathrm{N}$-glycans expressed by schistosomes, IgG subclass response against complex antennae schistosome glycans remain to be elucidated.

\section{AUTHOR CONTRIBUTIONS}

YY and KB performed experiments. YY, KB, and AvD analyzed data. AvD, N-CR, and CH designed and supervised the study. YY, $\mathrm{AvD}$, and $\mathrm{CH}$ wrote the paper.

\section{ACKNOWLEDGMENTS}

The authors thank Prof. David Dunne for making available the serum samples used in this study.

\section{SUPPLEMENTARY MATERIAL}

The Supplementary Material for this article can be found online at: https://www.frontiersin.org/articles/10.3389/fimmu. 2018.02331/full\#supplementary-material
Trop Med Int Health (2007) 12:431-44. doi: 10.1111/j.1365-3156.2006.01 805.x

4. Harn DA, Mitsuyama M, David JR. Schistosoma mansoni. Anti-egg monoclonal antibodies protect against cercarial challenge in vivo. J Exp Med. (1984) 159:1371-87.

5. McLaren DJ, Smithers SR. Serum from CBA/Ca mice vaccinated with irradiated cercariae of Schistosoma mansoni protects naive recipients through the recruitment of cutaneous effector cells. Parasitology (1988) 97 (Pt 2), 287-302.

6. Ko AI, Dräger UC, Harn DA. A Schistosoma mansoni epitope recognized by a protective monoclonal antibody is identical to the stage-specific embryonic antigen 1. Proc Natl Acad Sci USA. (1990) 87:4159-63. 
7. Attallah AM, Attia H, El-Nashar EM, Nawar A, Abdel Kader K, Ismail H, et al. Induction of resistance against Schistosoma mansoni infection by passive transfer of an IgG2a monoclonal antibody. Vaccine (1999) 17:2306-10.

8. Torben W, Ahmad G, Zhang W, Siddiqui AA. Role of antibodies in Smp80-mediated protection against Schistosoma mansoni challenge infection in murine and nonhuman primate models. Vaccine (2011) 29:2262-71. doi: 10.1016/j.vaccine.2011.01.040

9. Wuhrer M, Dennis RD, Doenhoff MJ, Lochnit G, Geyer R. Schistosoma mansoni cercarial glycolipids are dominated by Lewis $\mathrm{X}$ and pseudo-Lewis $\mathrm{Y}$ structures. Glycobiology (2000) 10:89-101. doi: 10.1093/glycob/10.1.89

10. Nyame AK, Lewis FA, Doughty BL, Correa-Oliveira R, Cummings RD. Immunity to schistosomiasis: glycans are potential antigenic targets for immune intervention. Exp Parasitol. (2003) 104:1-13. doi: 10.1016/S0014-4894(03)00110-3

11. Kariuki TM, Farah IO, Wilson RA, Coulson PS. Antibodies elicited by the secretions from schistosome cercariae and eggs are predominantly against glycan epitopes. Parasite Immunol. (2008) 30:554-62. doi: 10.1111/j.1365-3024.2008.01054.x

12. Luyai AE, Heimburg-Molinaro J, Prasanphanich NS, Mickum ML, Lasanajak Y, Song X, et al. Differential expression of anti-glycan antibodies in schistosome-infected humans, rhesus monkeys and mice. Glycobiology (2014) 24:602-18. doi: 10.1093/glycob/cwu029

13. Smit $\mathrm{CH}$, Kies CL, McWilliam HE, Meeusen EN, Hokke $\mathrm{CH}$, van Diepen A. Local antiglycan antibody responses to skin stage and migratory schistosomula of Schistosoma japonicum. Infect Immun. (2015) 84:21-33. doi: 10.1128/IAI.00954-15

14. van Diepen A, van der Plas AJ, Kozak RP, Royle L, Dunne DW, Hokke CH. Development of a Schistosoma mansoni shotgun O-glycan microarray and application to the discovery of new antigenic schistosome glycan motifs. Int J Parasitol. (2015) 45:465-75. doi: 10.1016/j.ijpara.2015.02.008

15. Yang YY, Li XH, Brzezicka K, Reichardt NC, Wilson RA, van Diepen A, et al. Specific anti-glycan antibodies are sustained during and after parasite clearance in Schistosoma japonicum-infected rhesus macaques. PLoS Negl Trop Dis. (2017) 11:e0005339. doi: 10.1371/journal.pntd.0005339

16. van Diepen A, Smit CH, van Egmond L, Kabatereine NB, Pinot de Moira A, Dunne DW, et al. Differential anti-glycan antibody responses in Schistosoma mansoni-infected children and adults studied by shotgun glycan microarray. PLoS Negl Trop Dis. (2012) 6:e1922. doi: 10.1371/journal.pntd.0001922

17. Bickle Q, Bain J, McGregor A, Doenhoff M. Factors affecting the acquisition of resistance against Schistosoma mansoni in the mouse: III. The failure of primary infections with cercariae of one sex to induce resistance to reinfection. Trans R Soc Trop Med Hyg. (1979) 73:37-41.

18. Bickle QD, Ford MJ, Andrews BJ. Studies on the development of antischistosomular surface antibodies by mice exposed to irradiated cercariae, adults and/or eggs of S. mansoni. Parasite Immunol. (1983) 5:499-511.

19. Eberl M, Langermans JA, Vervenne RA, Nyame AK, Cummings RD, Thomas AW, et al. Antibodies to glycans dominate the host response to schistosome larvae and eggs: is their role protective or subversive? J Infect Dis. (2001) 183:1238-47. doi: 10.1086/319691

20. Gong W, Huang F, Ma Y, Bai H, Yin L, Li J, et al. Protective immunity against Schistosoma japonicum infection can be provided by IgG antibodies towards periodate-sensitive or periodate-resistant glycans. Parasit Vectors (2015) 8:234. doi: 10.1186/s13071-015-0842-1

21. Brzezicka K, Echeverria B, Serna S, van Diepen A, Hokke CH, Reichardt NC. Synthesis and microarray-assisted binding studies of core xylose and fucose containing N-glycans. ACS Chem Biol. (2015) 10:1290-302. doi: 10.1021/cb501023u

22. Gaze S, Driguez P, Pearson MS, Mendes T, Doolan DL, Trieu A, et al. An immunomics approach to schistosome antigen discovery: antibody signatures of naturally resistant and chronically infected individuals from endemic areas. PLoS Pathog. (2014) 10:e1004033. doi: 10.1371/journal.ppat.1004033

23. Butterworth A, Dunne D, Fulford A, Capron M, Khalife J, Capron A, et al. Immunity in human schistosomiasis mansoni: cross-reactive IgM and IgG2 anti-carbohydrate antibodies block the expression of immunity. Biochimie (1988) 70:1053-63.

24. Hagan P, Blumenthal UJ, Dunn D, Simpson AJ, Wilkins HA. Human IgE, IgG4 and resistance to reinfection with Schistosoma haematobium. Nature (1991) 349:243-5. doi: 10.1038/349243a0
25. Khalife J, Dunne DW, Richardson BA, Mazza G, Thorne KJ, Capron A, et al. Functional role of human IgG subclasses in eosinophil-mediated killing of schistosomula of Schistosoma mansoni. J Immunol. (1989) 142:4422-7.

26. Boctor FN, Peter JB. IgG subclasses in human chronic schistosomiasis: overproduction of schistosome-specific and non-specific IgG4. Clin Exp Immunol. (1990) 82:574-8.

27. Iskander R, Das PK, Aalberse RC. IgG4 antibodies in Egyptian patients with schistosomiasis. Int Arch Allergy Appl Immunol. (1981) 66:200-7.

28. Amoah AS, Asuming-Brempong EK, Obeng BB, Versteeg SA, Larbi IA, Aryeetey $\mathrm{Y}$, et al. Identification of dominant anti-glycan IgE responses in school children by glycan microarray. J Allergy Clin Immunol. (2017). doi: 10.1016/j.jaci.2017.09.040

29. Naus CW, Booth M, Jones FM, Kemijumbi J, Vennervald BJ, Kariuki CH, et al. The relationship between age, sex, egg-count and specific antibody responses against Schistosoma mansoni antigens in a Ugandan fishing community. Trop Med Int Health (2003) 8:561-8. doi: 10.1046/j.1365-3156.2003.01056.x

30. Oyelaran O, McShane LM, Dodd L, Gildersleeve JC. Profiling human serum antibodies with a carbohydrate antigen microarray. J Proteome Res. (2009) 8:4301-10. doi: 10.1021/pr900515y

31. Smit $\mathrm{CH}$, van Diepen A, Nguyen DL, Wuhrer M, Hoffmann KF, Deelder $\mathrm{AM}$, et al. Glycomic analysis of life stages of the human parasite Schistosoma mansoni reveals developmental expression profiles of functional and antigenic glycan motifs. Mol Cell Proteomics (2015) 14:1750-69. doi: 10.1074/mcp.M115.048280

32. van Ree R, Cabanes-Macheteau M, Akkerdaas J, Milazzo JP, LoutelierBourhis C, Rayon C, et al. $\beta(1,2)$-xylose and $\alpha(1,3)$-fucose residues have a strong contribution in IgE binding to plant glycoallergens. J Biol Chem. (2000) 275:11451-8. doi: $10.1074 /$ jbc. 275.15 .11451

33. Altmann F. The role of protein glycosylation in allergy. Int Arch Allergy Immunol. (2007) 142:99-115. doi: 10.1159/000096114

34. Lee SI, Heiner DC, Wara D. Development of serum IgG subclass levels in children. Monogr Allergy (1986) 19:108-21.

35. Tran MH, Pearson MS, Bethony JM, Smyth DJ, Jones MK, Duke M, et al. Tetraspanins on the surface of Schistosoma mansoni are protective antigens against schistosomiasis. Nat Med. (2006) 12:835-40. doi: 10.1038/nm1430

36. van Die I, Gomord V, Kooyman FN, van den Berg TK, Cummings RD, Vervelde L. Core alpha1->3-fucose is a common modification of N-glycans in parasitic helminths and constitutes an important epitope for IgE from Haemonchus contortus infected sheep. FEBS Lett. (1999) 463:189-93.

37. Wuhrer M, Balog CI, Catalina MI, Jones FM, Schramm G, Haas H, et al. IPSE/alpha-1, a major secretory glycoprotein antigen from schistosome eggs, expresses the Lewis X motif on core-difucosylated N-glycans. FEBS J. (2006) 273:2276-92. doi: 10.1111/j.1742-4658.2006.05242.x

38. Meevissen MH, Wuhrer M, Doenhoff MJ, Schramm G, Haas H, Deelder AM, et al. Structural characterization of glycans on omega-1, a major Schistosoma mansoni egg glycoprotein that drives Th2 responses. J Proteome Res. (2010) 9:2630-42. doi: 10.1021/pr100081c

39. Meevissen MH, Balog CI, Koeleman CA, Doenhoff MJ, Schramm G, Haas H, et al. Targeted glycoproteomic analysis reveals that kappa-5 is a major, uniquely glycosylated component of Schistosoma mansoni egg antigens. Mol Cell Proteomics (2011) 10:M110 005710. doi: 10.1074/mcp.M110. 005710

40. Wilson IB, Harthill JE, Mullin NP, Ashford DA, Altmann F. Core alpha1,3fucose is a key part of the epitope recognized by antibodies reacting against plant N-linked oligosaccharides and is present in a wide variety of plant extracts. Glycobiology (1998) 8:651-61.

Conflict of Interest Statement: The authors declare that the research was conducted in the absence of any commercial or financial relationships that could be construed as a potential conflict of interest.

Copyright (C) 2018 Yang, van Diepen, Brzezicka, Reichardt and Hokke. This is an open-access article distributed under the terms of the Creative Commons Attribution License (CC BY). The use, distribution or reproduction in other forums is permitted, provided the original author(s) and the copyright owner(s) are credited and that the original publication in this journal is cited, in accordance with accepted academic practice. No use, distribution or reproduction is permitted which does not comply with these terms. 